\title{
Predictive and prognostic value of CT based radiomics signature in locally advanced head and neck cancers patients treated with concurrent chemoradiotherapy or bioradiotherapy and its added value to Human Papillomavirus status
}

Citation for published version (APA):

Ou, D., Blanchard, P., Rosellini, S., Levy, A., Nguyen, F., Leijenaar, R. T. H., Garberis, I., Gorphe, P., Bidault, F., Ferte, C., Robert, C., Casiraghi, O., Scoazec, J-Y., Lambin, P., Temam, S., Deutsch, E., \& Tao, Y. (2017). Predictive and prognostic value of CT based radiomics signature in locally advanced head and neck cancers patients treated with concurrent chemoradiotherapy or bioradiotherapy and its added value to Human Papillomavirus status. Oral Oncology, 71, 150-155.

https://doi.org/10.1016/j.oraloncology.2017.06.015

Document status and date:

Published: 01/08/2017

DOI:

10.1016/j.oraloncology.2017.06.015

Document Version:

Publisher's PDF, also known as Version of record

Document license:

Taverne

Please check the document version of this publication:

- A submitted manuscript is the version of the article upon submission and before peer-review. There can be important differences between the submitted version and the official published version of record. People interested in the research are advised to contact the author for the final version of the publication, or visit the DOI to the publisher's website.

- The final author version and the galley proof are versions of the publication after peer review.

- The final published version features the final layout of the paper including the volume, issue and page numbers.

Link to publication

\footnotetext{
General rights rights.

- You may freely distribute the URL identifying the publication in the public portal. please follow below link for the End User Agreement:

www.umlib.nl/taverne-license

Take down policy

If you believe that this document breaches copyright please contact us at:

repository@maastrichtuniversity.nl

providing details and we will investigate your claim.
}

Copyright and moral rights for the publications made accessible in the public portal are retained by the authors and/or other copyright owners and it is a condition of accessing publications that users recognise and abide by the legal requirements associated with these

- Users may download and print one copy of any publication from the public portal for the purpose of private study or research.

- You may not further distribute the material or use it for any profit-making activity or commercial gain

If the publication is distributed under the terms of Article $25 \mathrm{fa}$ of the Dutch Copyright Act, indicated by the "Taverne" license above,

Download date: 26 Apr. 2023 


\title{
Predictive and prognostic value of CT based radiomics signature in locally advanced head and neck cancers patients treated with concurrent chemoradiotherapy or bioradiotherapy and its added value to Human Papillomavirus status
}

\author{
Dan Ou ${ }^{\mathrm{a}, \mathrm{b}}$, Pierre Blanchard ${ }^{\mathrm{a}}$, Silvia Rosellini ${ }^{\mathrm{c}}$, Antonin Levy ${ }^{\mathrm{a}}$, France Nguyen ${ }^{\mathrm{a}}$, Ralph T.H. Leijenaar ${ }^{\mathrm{d}}$, \\ Ingrid Garberis ${ }^{\mathrm{e}}$, Philippe Gorphe ${ }^{\mathrm{f}}$, François Bidault ${ }^{\mathrm{g}}$, Charles Ferté ${ }^{\mathrm{f}}$, Charlotte Robert ${ }^{\mathrm{a}}$, Odile Casiraghi ${ }^{\mathrm{e}}$, \\ Jean-Yves Scoazec ${ }^{e}$, Philippe Lambin ${ }^{\mathrm{d}}$, Stephane Temam ${ }^{\mathrm{f}}$, Eric Deutsch ${ }^{\mathrm{a}}$, Yungan Tao ${ }^{\mathrm{a}, *}$
}

${ }^{a}$ Department of Radiation Oncology, Institut Gustave Roussy, Villejuif, France

${ }^{\mathrm{b}}$ Department of Radiation Oncology, Fudan University Shanghai Cancer Center, Shanghai, China

${ }^{\mathrm{c}}$ Department of Biostatistics, Institut Gustave Roussy, Villejuif, France

${ }^{\mathrm{d}}$ Department of Radiation Oncology, MAASTRO Clinic, Research Institute GROW, Maastricht University, 6229ET Maastricht, The Netherlands

e Department of Pathology, Institut Gustave Roussy, Villejuif, France

${ }^{\mathrm{f}}$ Department of Head and Neck Oncology, Institut Gustave Roussy, Villejuif, France

${ }^{\mathrm{g}}$ Department of Radiology, Institut Gustave Roussy, Villejuif, France

\section{A R T I C L E I N F O}

\section{Article history:}

Received 8 March 2017

Received in revised form 12 June 2017

Accepted 18 June 2017

Available online 26 June 2017

Keywords:

Radiomics

Locally advanced head and neck cancer HPV

Chemoradiotherapy

Bioradiotherapy

\begin{abstract}
A B S T R A C T
Objectives: To explore prognostic and predictive value of radiomics in patients with locally advanced head and neck squamous cell carcinomas (LAHNSCC) treated with concurrent chemoradiotherapy (CRT) or bioradiotherapy (BRT).

Materials and Methods: Data of 120 patients (CRT vs. BRT matched 2:1) were retrospectively analyzed. A total of 544 radiomics features of the primary tumor were extracted from radiotherapy planning computed tomography scans. Cox proportional hazards models were used to examine the association between survival and radiomics features with false discovery rate correction. The discriminatory performance was evaluated using receiver operating characteristic curve analysis.

Results: Multivariate analysis showed a 24-feature based signature significantly predicted for OS $(\mathrm{HR}=0.3, P=0.02)$ and progression-free survival $(\mathrm{PFS})(\mathrm{HR}=0.3, P=0.01)$. Combining the radiomics signature with p16 status showed a significant improvement of prognostic performance compared with p16 ( $A U C=0.78$ vs. $A U C=0.64$ at 5 years, $P=0.01$ ) or radiomics signature (AUC $=0.78$ vs. $A U C=0.67$, $P=0.01$ ) alone. When patients were stratified according to this combination, OS and PFS were significantly different according to the 4 sub-types (p16+ with low/high signature score; p16- with low/high signature score $)(P<0.001)$. Patients with high signature score significantly benefited from CRT (vs. BRT) in terms of OS $(P=0.004)$, while no benefit from CRT in patients with low signature score.

Conclusion: Our analysis suggests an added value of radiomics features as prognostic and predictive biomarker in HNSCC treated with CRT/BRT. Moreover, the radiomics signature provided additional information to HPV/p16 status to further stratify patients. External validation of such findings is mandatory given the risk of overfitting.
\end{abstract}

(c) 2017 Elsevier Ltd. All rights reserved.

\section{Introduction}

The current standard of care for patients with locally advanced head and neck squamous cell carcinoma (LAHNSCC) is concurrent

\footnotetext{
* Corresponding author at: Department of Radiation Oncology, Institut Gustave Roussy, 114 Rue Edouard Vaillant 94805 Villejuif France.

E-mail address: yungan.tao@gustaveroussy.fr (Y. Tao).
}

chemoradiotherapy (CRT), although it is associated with frequent severe acute and late toxicities [1]. In the recent years there has been a growing interest in increasing the benefit/risk ratio in human papillomavirus (HPV)-positive patients [2,3]. Due to improved prognosis, CRT could be safely postponed in HPV positive patients, reducing toxicity while not jeopardizing survival. Despite the overall good prognosis for HPV+ population, a substantial proportion of these patients remain at risk of recurrence and death [4]. Current recogni- 
tion of prognostic factors mainly based on tobacco exposure, TNM classification, performance status and comorbidities. Additional biomarkers are needed to further stratify patients and select candidates for studies testing de-intensification approaches.

Generally, tissue or blood sampling is required to evaluate most of the biomarkers. Invasive biopsies performed with that purpose are prone to sampling bias and may not be able to accurately reflect true heterogeneity of the entire tumor. Easily-obtainable, noninvasive prognostic biomarkers that allow assessment of tumor heterogeneity are lacking.

Inspired by the high-throughput success of the "omics", there has been resurgent interest in radiomics, which converts imaging data into a high dimensional mineable feature space, by using a large number of automatically extracted data-characterization algorithms [5]. Radiomics can provide a more comprehensive view of the entire tumor. Recent studies demonstrated that radiomics features had significant associations with clinical factors and underlying genomic patterns in various cancer types, thus showed strong prognostic performance [6-9]. In head and neck cancers, computed tomography (CT) imaging is routinely used for patient management, including diagnosis, radiation treatment planning and surveillance. Therefore, radiomics has significant clinical potential without adding additional imaging studies.

The purpose of this investigation was to develop a radiomics signature to estimate overall survival (OS) in patients with locally advanced head and neck squamous cell carcinomas (LAHNSCC) treated with concurrent chemoradiotherapy (CRT) or bioradiotherapy (BRT) and assess its incremental value to Human Papillomavirus (HPV) and clinical risk factors for individual OS estimation, and also to explore its predictive value.

\section{Materials and methods}

\section{Patients}

The retrospective cohort consisted of 120 patients with pathologically-confirmed head and neck squamous cell carcinoma (HNSCC), stage III-IVb according to American Joint Committee on Cancer (AJCC)/ International Union for Cancer Control (UICC) TNM classification 2010. Patients received total radiation dose of $70 \mathrm{~Gy}, \geq 2$ cycles of concurrent CDDP or $\geq 3$ cycles of concurrent cetuximab were selected from the former study cohort of 265 patients $[10,11]$. Patients were matched according to each $\mathrm{T}$ and $\mathrm{N}$ stages, with a 2:1 ratio of CRT vs. BRT patients. Institutional research ethics board approval was obtained.

All patients received either one of the following two treatments between June 2006 and October 2012: definitive radiotherapy (RT) concomitantly with cisplatin $\left(100 \mathrm{mg} / \mathrm{m}^{2}\right.$ every 3 weeks on days 1,22 , and 43 ) or cetuximab (initial loading dose of $400 \mathrm{mg} /$ $\mathrm{m}^{2}$ one week prior to RT, followed by weekly injection at $250 \mathrm{mg} /$ $\mathrm{m}^{2}$ during RT). Patients received either three-dimensional conformal radiotherapy (3D-CRT) or intensity-modulated radiotherapy (IMRT). External beam definitive RT was delivered with a total dose of $70 \mathrm{~Gy}$ to the gross tumor volume (GTV) in 35 fractions (range 3035 fractions) at 5 fractions per week, with median overall treatment time of 49 days (range 39-70 days). A dose of 60 Gy and 50-54 Gy were delivered to the intermediate- and low-risk clinical target volume (CTV). The CTVs were each expanded using 3-5 mm margins to generate their respective planning target volumes (PTV). Patient assessments in follow-up were previously described [10].

\section{CT imaging, image segmentation and radiomics analysis}

Planning CT of radiotherapy was performed according to standard clinical scanning protocols at Gustave Roussy with a Siemens
Somatom Sensation Open 24-slice scanner (Siemens Medical Solutions, Forchheim, Germany). The most common pixel spacing was (0.98 mm, $0.98 \mathrm{~mm}, 3 \mathrm{~mm}$ ) for CT.

The GTV of primary tumor (GTV-T) as region of interest was manually contoured on all CT slices by a radiation oncologist with expertise in identifying head and neck cancers. The segmentation was performed by using the treatment planning system (TPS) of ISOGRAY (DOSIsoft, Cachan, France).

The images and structures were exported from TPS in DICOM (Digital Imaging and Communications in Medicine) format. All image analysis was performed with the Oncoradiomics ${ }^{\mathrm{TM}}$ research software using Matlab R2012b (Oncoradiomics.com) [6]. In total, 544 radiomics image features were defined and were divided in four groups: (I) tumor intensity, (II) shape, (III) texture and (IV) wavelet features. Detailed definitions of all features and the extraction methods were previously described [6].

\section{Pathology}

Three $\mu \mathrm{m}$ thick sections were cut from the paraffin-embedded pretreatment biopsy specimens. HPV status was determined by p16 expression staining with immunohistochemistry (IHC). We considered p16 as positive when nuclear staining was $>75 \%$ of cells. Cytoplasmic only staining was considered as negative. All assessments were performed by the same experienced pathologist, who was blinded to the clinical and follow-up data.

\section{Statistical and bioinformatic analysis}

Survival rates were calculated with the Kaplan-Meier method. Survival times were defined as the time from the beginning of radiotherapy until either the time of first event or the date that the patient was last known to be alive (censored). Events were death from any cause for OS, death or tumor progression for progression-free survival (PFS), death or locoregional recurrence for loco-regional control (LRC), and death or distant metastasis for distant metastasis-free survival (DMFS). Survival curves were compared using the log-rank test.

Hierarchical clustering was applied to reveal the grouping patterns of patients. The correlation between the radiomics feature clusters and clinicopathologic variables were analyzed by chisquare test. Both traditional prognostic factors and radiomics features underwent univariate Cox proportional hazards model (CPHM) analysis. To adjust for multiple comparisons, a false discovery rate (FDR) correction was performed for radiomics features, and the FDR-corrected $p$ values were calculated using the Benjamini and Hochberg method. The radiomics features selected as candidate indicators under UVA were conducted with the principal component analysis (PCA). Variables with a $p$ value $<0.1$ on UVA entered the multivariate Cox regression analysis (MVA). In the Cox model, continuous variables (PS, T, N, Charlson index, age and radiomics signature score) were dichotomized. To allow appropriate dichotomization, the Youden index was utilized to choose a suitable cutoff point for the radiomics signature score with optimum sensitivity and specificity according to receiver operating characteristic curve (ROC) for predicting 5-year overall survival. The robustness of prognostic performance of the radiomic signature was assessed using 10 -fold cross validation by dichotomizing patients according to the survival or death status at 5 years (censored subjects within 60 months were excluded). Two-sided $P$ values $<0.05$ were considered to indicate a significant difference. The above analyses were performed using SPSS version 18.0 (SPSS Inc., Chicago, IL, USA) and R software version 3.2.4. 


\section{Results}

\section{Patient characteristics}

Patients' clinical and pathological characteristics are summarized in eTable 1 in Supplement. In total, HPV/p16 status of 101 patients had been determined by IHC. There were no significant distribution differences between CRT and BRT group except for the p16 status distribution.

\section{Survival and relapse}

The median follow-up time was of 49.3 months (range 3.7103.9 months). The 3-year actuarial OS, PFS and LRC for the entire population were $72.5 \%, 61.2 \%$, and $72.1 \%$, respectively. The 5-year actuarial OS, PFS and LRC were $61.2 \%, 52.6 \%$, and $72.1 \%$, respectively.

\section{Hierarchical clustering}

The hierarchical clustering of 120 patients based on radiomics feature values is shown in Fig. 1. Unsupervised clustering revealed 4 clusters of patients with similar radiomics expression patterns. We compared the 4 clusters of patients with clinical parameters, and found significant correlation with $\mathrm{T}$ classification $\left(P=3.3 * 10^{-5}\right)$, primary tumor location $(P=0.01)$ and a nonsignificant correlation with smoking status $(P=0.09)$, whereas no correlation was observed with p16 status $(P=0.19)$ and N classification $(P=0.22)$.

\section{Identification of potential prognostic radiomics features and definition of signature}

UVA evaluating the correlation of radiomics features and OS was performed for each of the 544 radiomics features. A total of
24 features remained statistically significant after FDR correction $(P=0.045)$, of which 3 were intensity features, 6 were shape features, 1 was texture features and 15 were wavelet features (eTable 2 in Supplement). PCA was used for the 24 features. Three principal components accounted for $87.6 \%$ (58.3\%, $23.0 \%$, and $6.3 \%$, respectively) of the variance. A radiomics signature was developed from the first principal component. The factor loadings are given in eTable 3 in Supplement.

\section{Prognostic value of the radiomics signature}

We classified the patients into high-score or low-score group using the optimal cutoff point of radiomics signature scores. The radiomics signature showed prognosis capacity for predicting 5year survival in the whole population with an AUC of 0.67 (95\% CI, 0.58-0.76) (eFig. 1 in Supplement). MVA adjusted for clinicopathological factors showed that both the radiomics signature score and p16 significantly predicted for OS (signature score: $\mathrm{HR}=0.3,95 \% \mathrm{CI}, 0.1-0.9, P=0.02 ; \mathrm{p} 16: \mathrm{HR}=0.3,95 \% \mathrm{CI}, 0.1-0.9$, $P=0.03$ ) and PFS (signature score: $\mathrm{HR}=0.3,95 \% \mathrm{CI}, 0.1-0.8$, $P=0.01 ;$ p16: $\mathrm{HR}=0.4,95 \% \mathrm{CI}, 0.2-1.1, P=0.08$ ) (Table 1 ). Compared with patients with high scores, patients with low scores had significantly better OS and PFS (eFig. 2 in Supplement). Spearman correlation showed no significant correlation between the radiomics signature score and p16 status (rho $=0.06, P=0.54$ ). The spearman correlation coefficient between smoking status, $\mathrm{T}$ classification, $\mathrm{N}$ classification and $\mathrm{PS}$ with radiomics signature score were $0.23(P=0.01), 0.41(P<0.001), 0.02(P=0.81), 0.30$ $(P<0.001)$, respectively.

The patients were then divided into 4 sub-types: p16+ and low signature score (PL); p16+ and high signature score (PH); p16- and low signature score (NL) and finally p16- and high signature score (NH). The AUC point estimates for this signature that incorporates radiomics score and p16 status was significantly superior to radiomics signature score or p16 alone throughout follow-up (Fig. 2a).

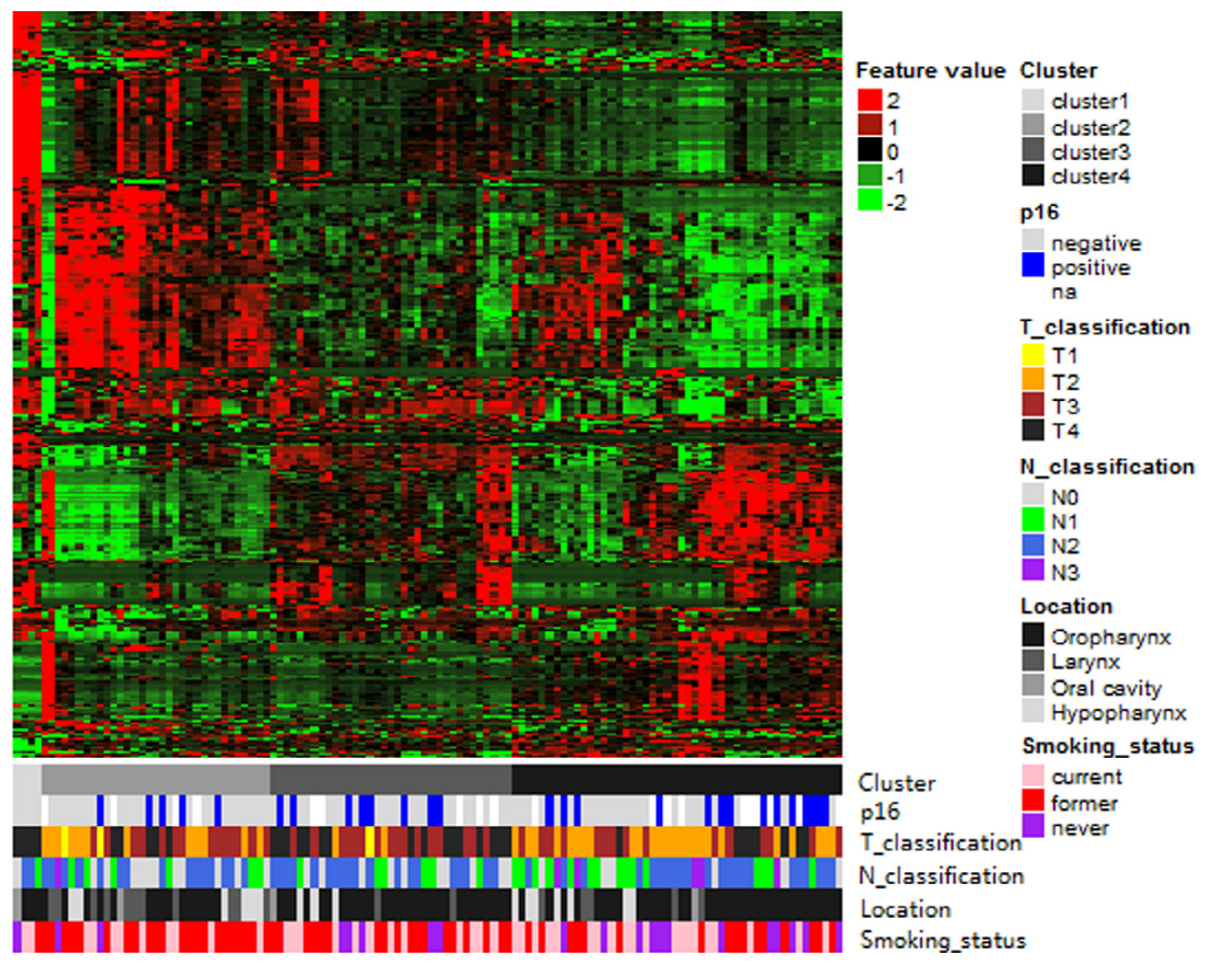

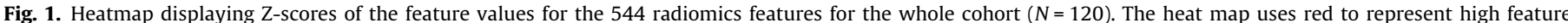

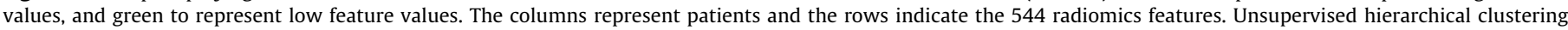
showed 4 clusters of patients with similar radiomics expression patterns. In the bottom color bars each color represents a specific class of sub-set. 
Table 1

Univariate and multivariate analyses of overall survival and progression-free survival $(N=120)$.

\begin{tabular}{|c|c|c|c|c|c|c|c|c|c|c|}
\hline \multirow[t]{3}{*}{ Variable } & \multicolumn{5}{|l|}{ OS } & \multicolumn{5}{|l|}{ PFS } \\
\hline & \multicolumn{2}{|l|}{ UVA } & \multicolumn{3}{|c|}{ MVA $^{a}$} & \multicolumn{2}{|c|}{ UVA } & \multicolumn{3}{|c|}{ MVA $^{a}$} \\
\hline & $\mathrm{HR}$ & $P$ & $\mathrm{HR}$ & $95 \% \mathrm{CI}$ & $P$ & $\mathrm{HR}$ & $P$ & $\mathrm{HR}$ & $95 \% \mathrm{CI}$ & $P$ \\
\hline T3-4 classification (T1-2) & 1.34 & 0.37 & 0.9 & $0.4-2.2$ & 0.84 & 1.0 & 0.90 & 0.7 & $0.3-1.6$ & 0.45 \\
\hline $\mathrm{N} 2-3$ classification (N0-1) & 1.8 & 0.08 & 1.3 & $0.6-2.8$ & 0.46 & 2.2 & 0.01 & 1.7 & $0.8-3.3$ & 0.16 \\
\hline Never smoker (Current/Former smoker) & 0.3 & 0.03 & 0.6 & $0.1-2.5$ & 0.46 & 0.2 & 0.01 & 0.4 & $0.3-1.6$ & 0.28 \\
\hline $\mathrm{BRT}(\mathrm{CRT})$ & 1.5 & 0.19 & & & & 1.5 & 0.13 & & & \\
\hline $\mathrm{p} 16+(\mathrm{p} 16-)$ & 0.2 & 0.01 & 0.3 & $0.1-0.9$ & 0.03 & 0.3 & 0.02 & 0.4 & $0.2-1.1$ & 0.08 \\
\hline $\mathrm{PS}=0(\mathrm{PS} \geq 1)$ & 0.5 & 0.03 & 0.9 & $0.4-1.8$ & 0.68 & 0.4 & 0.003 & 0.8 & $0.4-1.5$ & 0.46 \\
\hline Oropharynx (Non-oropharynx) & 1.0 & 0.89 & & & & 0.9 & 0.77 & & & \\
\hline IMRT (no-IMRT) & 0.3 & 0.11 & & & & 0.4 & 0.09 & 0.5 & $0.2-1.9$ & 0.34 \\
\hline Charlson index $\geq 2(0-1)$ & 1.2 & 0.56 & & & & 1.4 & 0.25 & & & \\
\hline Age $\geq 65$ years $(<65)$ & 1.5 & 0.25 & & & & 1.4 & 0.27 & & & \\
\hline Male (Female) & 1.2 & 0.68 & & & & 1.6 & 0.22 & & & \\
\hline Radiomics signature score low (high) & 0.4 & 0.004 & 0.3 & $0.1-0.9$ & 0.02 & 0.4 & 0.001 & 0.3 & $0.1-0.8$ & 0.01 \\
\hline
\end{tabular}

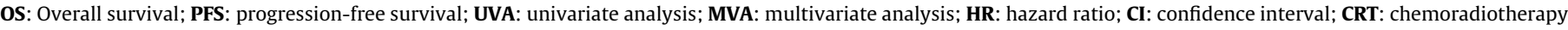
with concurrent cisplatin; BRT: bioradiotherapy with concurrent cetuximab; IMRT: intensity-modulated radiotherapy; PS: performance status.

a $N=101$.

When patients were stratified according to the radiomics signature score + p16, OS and PFS curves were significantly different according to the 4 sub-types ( $\mathrm{p}<0.001$ ). OS and PFS of the PL and NL subgroups were both significantly higher than their high signature score-counterparts (OS: $P=0.03, P=0.003$; PFS: $P=0.01$, $P=0.002$, respectively) (Fig. $2 \mathrm{~b}, \mathrm{c}$ ).

The prognostic performance of the clinical factors (smoking status, T, N classification and PS) which were significant in UVA for OS were also analyzed. eTable 4 in Supplement shows AUC estimates of 5-year OS of these factors alone and in combination with the radiomics signature. All the AUC estimates of the 4 factors improved when combining together with the radiomics signature compared to the factors alone. However, the combination with these factors didn't significantly improve AUC compared to the radiomics signature alone. The combination of radiomics signature and p16 status demonstrated the highest AUC estimate $(0.78,95 \%$ CI 0.68-0.88) among all the factors and combinations. According to the 10 -fold cross validation, the accuracy of radiomic signature prognostic model for 5 -year OS was 0.67 , and 0.74 of radiomic signature +p16 prognostic model (Supplementary eTable 4).

\section{Predictive value of the radiomics signature}

The 5-year OS of the CRT and BRT groups were $62.1 \%$ and $57.5 \%$, respectively $(P=0.20)$. The 5 -year PFS of the two groups were $54.4 \%$ and $47.2 \%$, respectively $(P=0.13)$. No significant differences of OS and PFS were observed between the two treatment groups (eFig. 3 in Supplement). However, when patients were further stratified by radiomics signure score, patients with high signature score significantly benefited more from CRT (vs. BRT) in terms of OS $(P=0.004)$ and PFS $(P=0.001)$, while no benefit difference between CRT and BRT in patients with low signature score $(P=0.99, P=0.90$, respectively) (Fig. 3).

\section{Discussion}

The utilization of imaging features is evolving from qualitative interpretation to more sophisticated quantitative analysis-radiomics, to non-invasively capture tumor phenotypic differences. CT imaging features of tumor heterogeneity have been associated with OS in patients with head and neck cancers, esophageal cancer, colorectal cancer, and non-small cell lung cancer (NSCLC) among others $[6,12-14]$.
Biomarker discovery with HNSCC is relatively limited. Although HPV status is deemed to be the strongest prognostic marker in HNSCC patients [15], to date, the standard treatment for HNSCC is not different between HPV positive and HPV negative tumors. Studies show that there is a small subgroup of HPV+ oropharyngeal cancer patients with poor prognosis and they need to be identified with more specific and selective markers [16]. The search for additional methods is ongoing, mainly based on smoking history and tumor-infiltrating lymphocytes [17,18]. Meanwhile, for patients with HPV-negative HNSCC, few prognostic factors other than TNM classification, surgical margins, and extracapsular infiltration has been identified. Novel, non-invasive biomarkers with high prognostic and predictive performance are needed for risk stratification and treatment personalization. We identified a 24-feature signature as an independent predictor for HNSCC patients' OS and PFS.

CT imaging feature quantification of tumor heterogeneity has been associated with HPV status of HNSCC. In the two analyses of Sakai et al. [19,20], statistically significant differences in some texture features between HPV+ and HPV - HNSCC have been found. However, a single radiomics feature may not have enough predictive power. Parmar et al. identified different Lung and H\&N radiomics cancer-specific feature subgroups and quantified their clinical significance by clustering, combining clinical, imaging and gene expression data with external validation sets [21]. Similar to the study of Aerts et al. [6], our clustering analysis revealed different clusters of patients with similar radiomics expression patterns, and found significant correlation of radiomics clusters with $\mathrm{T}$ classification, primary tumor location and a non-significant correlation with smoking status, which included the information of most of the traditional prognostic clinical factors. In addition, recently it is also reported that a 4-feature based radiomics signature showed strong correlation with survival and distant metastasis across different cohorts and different types of cancers (NSCLC and HNSCC). The concordance index $(\mathrm{CI})$ in the training cohorts and validation cohorts were ranging from 0.55 to 0.69 [6,9,22]. The AUC of the ROC curve of our radiomics signature predicting 5-year OS was 0.67 (95\%CI, 0.58-0.76), which was similar to the previous reported results.

To the authors' knowledge, the current study is the first assessing the combining prognostic value of radiomics signature and $\mathrm{p} 16$, and also the first exploring the predictive value of radiomics in patients with LAHNSCC treated with CRT or BRT. The combination showed superior improved predictive accuracy of OS compared to p16 alone. Further, we demonstrated that the radiomics signature 

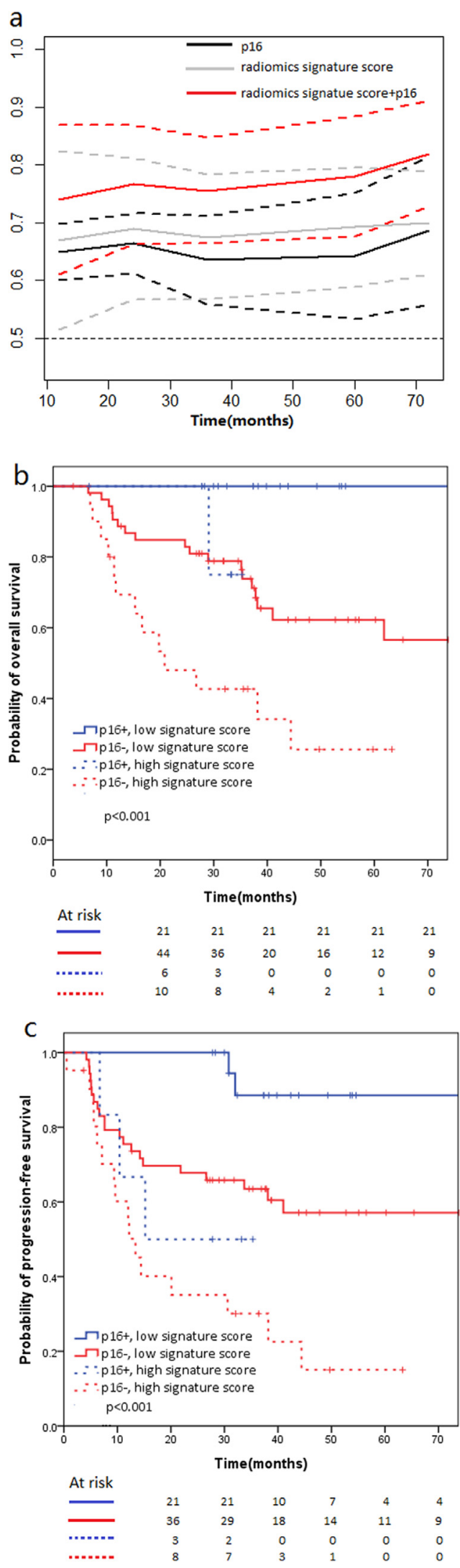

Fig. 2. (a) Time-dependent AUC estimates through follow-up when applying 3 prognostic biomarkers. The regions between the two dashed lines of the same color provide a 95\% confidence interval for the corresponding AUC. (b) Kaplan-Meier curves of overall survival for patients stratified by radiomics signature score and p16: PL vs. PH, $P 1=0.03$; NL vs. NH, $P 2=0.003$. (c) Progression-free survival: PL vs. $\mathrm{PH}, \mathrm{P} 3=0.01 ; \mathrm{NL}$ vs. NH, $P 4=0.002$.
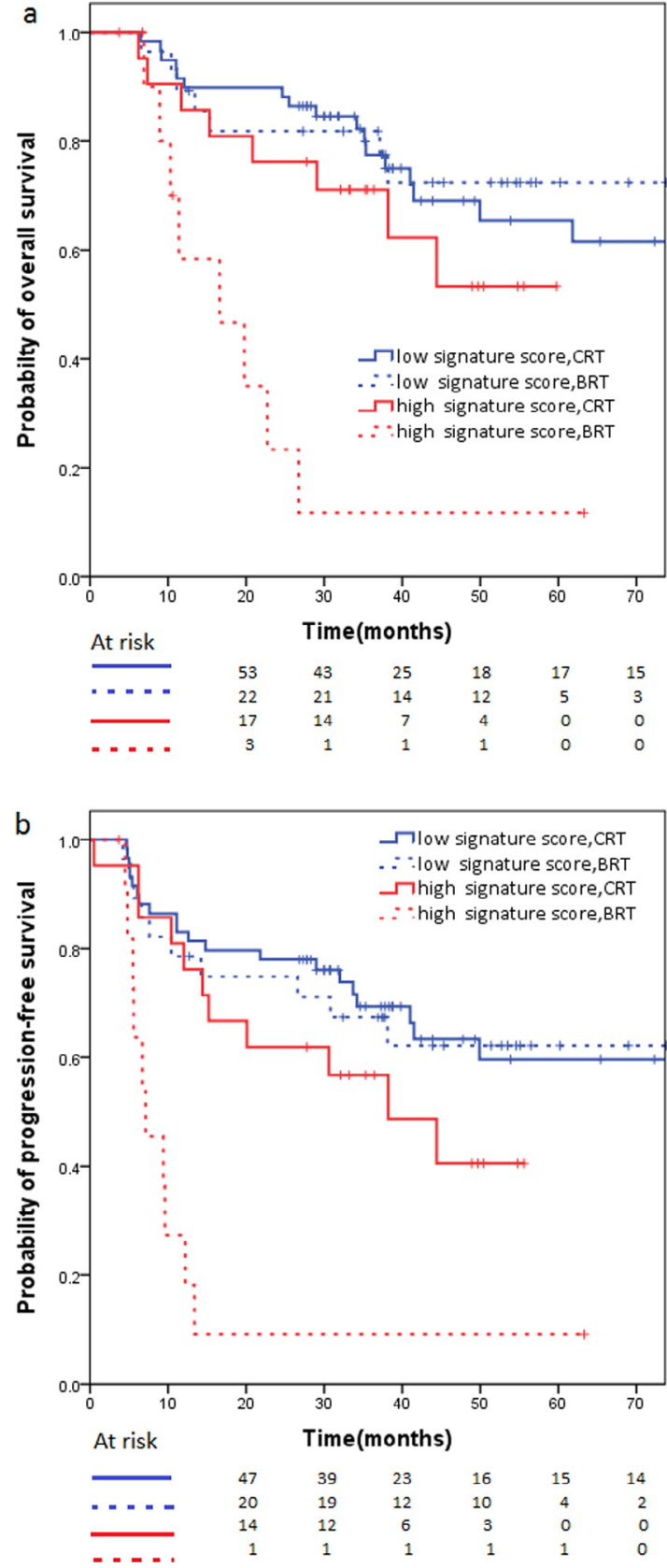

Fig. 3. Kaplan-Meier curves for patients stratified by radiomics signature score and concurrent treatment regimen. (a) Overall survival: low signature score, CRT vs. low signature score, BRT, $P 1=0.99$; high signature score, CRT vs. high signature score, BRT, $P 2=0.004$; (b) Progression-free survival: low signature score, CRT vs. low signature score, $B R T, P 3=0.90$; high signature score, $C R T$ vs. high signature score BRT, $P 4=0.001$

can potentially stratify sub-groups of HPV+ and HPV- HNSCC patients. This characterization of sub-population in addition of HPV status for LAHNSCC patients might guide future treatment selection, which would lead to improvement of quality of life and/or survival.

Although evidences are emerging comparing CRT versus BRT for treating LAHNSCC, so far published results are controversial [23]. Characterization of the optimal selection of patients benefiting from concurrent cisplatin or cetuximab has yet to be defined. In the study of Ou et al. [11], the benefit of CRT (vs BRT) were similar in $\mathrm{p} 16+$ and $\mathrm{p} 16-/ \mathrm{unknown}$ populations. Meanwhile, our results showed superior survival results using CRT compared to BRT in 
the sub-population of patients with high radiomics signature score, while in patients with low signature score, the two concurrent regimens showed no difference on treatment outcomes. In the study of Aerts et al. the intratumour heterogeneity radiomics features have been correlated with cell cycling pathways [6]. Thus the underlying mechanism of our results could be that the antiproliferative drug--CDDP was more efficient for the tumors with increased proliferation. These initial results implied that radiomics may play a role in treatment selection.

Our current study has several limitations, and should be considered as a preliminary study with need for independent external validation. It is a retrospective study with a relatively small population. The characteristics of the enrolled population were heterogeneous and the selection of treatment might be biased, so that the performance of the heterogeneity of features can be underestimated. Further, our dataset includes patients from 2006 to 2012. During this time period, the standard of care for CT acquisition has evolved, which could potentially affect our analysis. Additionally, HPV status was determined using p16 expression staining with IHC, which is only a surrogate in the oropharynx, whereas in our study other tumor sites were also included. No combining tests (In situ Hybridization or polymerase chain reaction) for HPV validation was performed. Nowadays imaging examinations routinely performed in clinical practice for HNSCC include multiple modalities, such as CT, MRI, PET/CT, etc. An integrated radiomics analysis of different imaging modalities could potentially provide more information. It is also reported recently that radiomics is able to identify different gene expressions [6,24], and the correlation of radiomics with other potential biomarkers such as PD-1/PD-L1 is not evaluated in our current analysis. In fact a parallel study of molecular biomarkers in the same population is under planning to further investigate the correlation of radiomics and other valuable biomarkers. Future work would also involve studying the signature in independent validation sets from other institutions.

\section{Conclusion}

In the present study, we investigated the correlation of radiomics signature with survival and its predictive potential in LAHNSCC patients treated with concurrent chemoradiotherapy or bioradiotherapy. The signature had an additional discrimination capability when added to p16 status, improving the prognostic accuracy and allowing further stratifying patients according to their risk of relapse. While these results require external validation (ongoing), they add to the growing body of evidence that radiomics quantification non-invasively predicts patients' outcome and may in the future be used to guide treatment selection.

\section{Role of the funding source}

This work was supported by EU 7th framework program (ARTFORCE), No Grant No. is applicable; and China Scholarship Council (CSC) [201406105021 to D.O.]. This research was also supported by the Dutch Technology Foundation STW (grant $n^{\circ} 10696$ DuCAT \& $n$ - P14-19 Radiomics STRaTegy). Authors also acknowledge financial support from the European Program H2020-PHC-2015 (BD2Decide - PHC30-689715 and Alpe d'HuZes-KWF (DESIGN). We thank the company Oncoradiomics (www.oncoradiomics.com) for having provided gracefully the oncoradiomics ${ }^{\mathrm{TM}}$ research software.

\section{Conflict of interest statement}

\section{Appendix A. Supplementary material}

Supplementary data associated with this article can be found, in the online version, at http://dx.doi.org/10.1016/j.oraloncology. 2017.06.015.

\section{References}

[1] Pignon JP, le Maître A, Maillard E, et al. Meta-analysis of chemotherapy in head and neck cancer (MACH-NC): an update on 93 randomised trials and 17,346 patients. Radiother Oncol 2009;92:4-14.

[2] Rietbergen MM, Witte BI, Velazquez ER, et al. Different prognostic models for different patient populations: validation of a new prognostic model for patients with oropharyngeal cancer in Western Europe. $\mathrm{Br} \mathrm{J}$ Cancer 2015;112:1733-6.

[3] Rios Velazquez E, Hoebers F, Aerts HJ, et al. Externally validated HPV-based prognostic nomogram for oropharyngeal carcinoma patients yields more accurate predictions than TNM staging. Radiother Oncol 2014;113:324-30.

[4] Quon H, Forastiere AA. Controversies in treatment deintensification of human papillomavirus-associated oropharyngeal carcinomas: should we, how should we, and for whom? J Clin Oncol 2013;31:520-2.

[5] Lambin P, Rios-Velazquez E, Leijenaar R, et al. Radiomics: extracting more information from medical images using advanced feature analysis. Eur J Cancer 2012;48:441-6.

[6] Aerts HJ, Velazquez ER, Leijenaar RT, et al. Decoding tumour phenotype by noninvasive imaging using a quantitative radiomics approach. Nat Commun 2014;5:4006.

[7] Yip C, Landau D, Kozarski R, et al. Primary esophageal cancer: heterogeneity as potential prognostic biomarker in patients treated with definitive chemotherapy and radiation therapy. Radiology 2014;270:141-8.

[8] Pickles M, Lowry M, Gibbs P. Pretreatment prognostic value of dynamic contrast-enhanced magnetic resonance imaging vascular, texture, shape, and size parameters compared with traditional survival indicators obtained from locally advanced breast cancer patients. Invest Radiol 2016;51:177-85.

[9] Leijenaar RT, Carvalho S, Hoebers FJ, et al. External validation of a prognostic CT-based radiomic signature in oropharyngeal squamous cell carcinoma. Acta Oncol 2015;54:1423-9.

[10] Levy A, Blanchard P, Bellefqih S, et al. Concurrent use of cisplatin or cetuximab with definitive radiotherapy for locally advanced head and neck squamous cell carcinomas. Strahlenther Onkol 2014:190:823-31.

[11] Ou D, Levy A, Blanchard P, et al. Concurrent chemoradiotherapy with cisplatin or cetuximab for locally advanced head and neck squamous cell carcinomas: does human papilloma virus play a role? Oral Oncol 2016;59:50-7.

[12] Ganeshan B, Skogen K, Pressney I, et al. Tumour heterogeneity in oesophageal cancer assessed by CT texture analysis: preliminary evidence of an association with tumour metabolism, stage, and survival. Clin Radiol 2012;67:157-64.

[13] Ng F, Ganeshan B, Kozarski R, et al. Assessment of primary colorectal cancer heterogeneity by using whole-tumor texture analysis: contrast-enhanced CT texture as a biomarker of 5-year survival. Radiology 2013;266:177-84.

[14] Zhang H, Graham CM, Elci O, et al. Locally advanced squamous cell carcinoma of the head and neck: CT texture and histogram analysis allow independent prediction of overall survival in patients treated with induction chemotherapy. Radiology 2013;269:801-9.

[15] Lassen P. The role of Human papillomavirus in head and neck cancer and the impact on radiotherapy outcome. Radiother Oncol 2010;95:371-80.

[16] Ang KK, Harris J, Wheeler R, et al. Human papillomavirus and survival of patients with oropharyngeal cancer. N Engl J Med 2010;363:24-35.

[17] Bhatia A, Burtness B. Human papillomavirus-associated oropharyngeal cancer: defining risk groups and clinical trials. J Clin Oncol 2015;33:3243-50.

[18] Badoual C, Hans S, Merillon N, et al. PD-1-expressing tumor-infiltrating T Cells are a favorable prognostic biomarker in HPV-associated head and neck cancer. Can Res 2013;73:128-38.

[19] Buch K, Fujita A, Li B, et al. Using texture analysis to determine human papillomavirus status of oropharyngeal squamous cell carcinomas on CT. Am J Neuroradiol 2015;36:1343-8.

[20] Fujita A, Buch K, Li B, et al. Difference between HPV-positive and HPV-negative non-oropharyngeal head and neck cancer: texture analysis features on CT. J Comput Assist Tomogr 2016;40:43-7.

[21] Parmar C, Leijenaar RT, Grossmann P, et al. Radiomic feature clusters and Prognostic Signatures specific for Lung and Head \& Neck cancer. Sci Rep 2015;5:11044

[22] Coroller TP, Grossmann P, Hou Y, et al. CT-based radiomic signature predicts distant metastasis in lung adenocarcinoma. Radiother Oncol 2015; 114:345-50.

[23] Husain ZA, Burtness BA, Decker RH. Cisplatin versus cetuximab with radiotherapy in locally advanced squamous cell carcinoma of the head and neck. J Clin Oncol 2016;34:396-8.

[24] Panth KM, Leijenaar RT, Carvalho S, et al. Is there a causal relationship between genetic changes and radiomics-based image features? An in vivo preclinical experiment with doxycycline inducible GADD34 tumor cells. Radiother Oncol 2015;116:462-6.

None declared. 